\title{
Dynamique d'incorporation du carbone et des éléments nutritifs dans un taillis simple de châtaignier (Castanea sativa Miller)
}

\author{
J Ranger $1^{*}$, C Félix 1, J Bouchon 2, C Nys 1, M Ravart 2 \\ avec la collaboration de $\mathrm{P}$ Bonnaud 1, D Bouchard 1, C Bréchet 1, \\ R Canta ${ }^{2}$, P Garnier 2, L Gelhaye 1, D Delhaye ${ }^{1}$ \\ 1 INRA-CRF Nancy, station de recherche sur le sol, la microbiologie \\ et la nutrition des arbres forestiers, Champenoux, 54280 Seichamps; \\ 2 INRA-CRF Nancy, station de sylviculture et production, Champenoux 54280 Seichamps, France
}

(Reçu le 6 février 1990; accepté le 15 mars 1990)

\begin{abstract}
Résumé - Les travaux effectués sur les taillis simples de châtaignier ont pour objectif de fournir des données sur les immobilișations d'éléments nutritifs en fonction de la fertilité de la station ou de la longueur de la rotation. Ces études appartiennent à des recherches plus générales sur l'effet de l'intensification de la sylviculture des taillis simples sur l'exportation d'éléments nutritifs et par conséquent, sur le maintien de la fertilité des sols dans ce contexte. Le modèle châtaignier a été utilisé pour étudier la dynamique d'incorporation des éléments nutritifs au cours du cycle sylvicole; pour ceci une série de 5 peuplements d'âge croissant, situés dans un même contexte écologique et ayant subi le même traitement sylvicole sont étudiés quant à leur production de biomasse et leur contenu en éléments nutritifs. La construction de tarifs de biomasse et de minéralomasse, applicables à la série d'âge de peuplements a été testée. Les résultats montrent que le paramétrage par l'âge des tarifs de biomasse est toujours nécessaire, alors que c'est rarement le cas pour la minéralomasse. Dans un prochain travail nous examinerons en détail les variations précises des éléments avec l'âge, afin de déterminer, en particulier, la participation des transferts internes d'éléments nutritifs (cycle biochimique) dans la nutrition des peuplements. Dans le contexte écologique en question, les taillis simples de châtaignier peuvent produire $7,2 \mathrm{t}$ de matière sèche par ha et par an à 15 ans. C'est vraisemblablement la production maximale que l'on peut espérer obtenir dans ces taillis d'ensouchement ancien. L'étude de la dynamique des peuplements montre que la vitesse d'incorporation d'éléments nutritifs diminue considérablement avec l'âge; la vitesse courante d'incorporation est même nulle voire négative entre 15 et 19 ans, ce qui signifie que la production est gratuite pour l'écosystème entre ces 2 âges. Cette approche permet de chiffrer les restitutions totales au sol (iitière + nécromasse). Les mécanismes de recyclage interne d'éléments nutritifs et de restitutions et de minéralisation rapide des litières constituent une stratégie efficace d'indépendance des peuplements vis-à-vis des sols et autorisent une croissance importante dans des contextes de sols de fertilité souvent réduite. Le châtaignier, compte tenu de sa production et de la qualité des produits, devrait pouvoir participer à des scénarios de boisement des terres libérées par l'agriculture.
\end{abstract}

châtaignier / taillis / biomasse / minéralomasse / dynamique de développement

\footnotetext{
* Correspondance et tirés à part
} 
Summary - Dynamics of carbon and nutrient accumulation in a coppiced chestnut (Castanea sativa Miller) stand. The aim of the research on chestnut coppices was to gain data on the nutrient accumulation in the stands as a function of soil fertility, harvest intensity and rotation length. This research forms part of a more general programme on the effects of sylviculture intensification in the coppice system, on the export of nutrients from forest soils and consequently on the maintenance of soil fertility. Chestnut was used in this work to study the dynamics of carbon and nutrient accumulation during stand development. As the ideal method of studying a single stand was not possible, an age series of stands was used. The major constraints of this approach are related to soil heterogeneity and identity of the silvicultural treatments in all the stands. The construction of biomass and nutrient content tables applicable to the stands of different ages were tested. The results showed that for biomass measurements, it was necessary to use age as a parameter for the coefficients of regressions for all the stand components; for the nutrient content, the incorporation of age in the equations was very seldom necessary. It is clear that these differences are linked to the characteristics of carbon and nutrients in the vegetation: carbon is fixed definitively in the perennial components of trees while the nutrients are recycled from the old to the young tissues. Under the ecological conditions of the site studied (mesotrophic to eutrophic brown soil), the chestnut stand produced $7.2 t$ of dry mater per ha per year at 15 yr old. This production is probably the greatest that can be found in coppices with numerous rotations. The study of stand dynamics showed that the nutrient incorporation rate decreased considerably with stand ageing; the current rate equals zero between 15 and $19 \mathrm{yr}$ old. This means that the production is cost-free for the ecosystem at this stage of stand development. This approach allows us to evaluate the total return of litter to the soil (litter ss and tree mortality). For the stands, internal recycling, the importance of total return and the rate of humus, mineralization constituted an efficient strategy as regards independence from soil nutrient reserves, and permit a significant growth in the content of soils with reduced fertility. Considering the production and the wood quality of chestnut stands, this species could be used for projected afforestation of land abandoned by agriculture.

chestnut tree / coppice / biomass / nutrient content / stand development dynamics

\section{INTRODUCTION}

Le châtaignier (Castanea sativa Miller) est une espèce d'origine asiatique, anciennement et largement introduite en France dans le bassin méditerranéen puis dans une grande partie du territoire, soit pour la culture fruitière, soit pour le bois.

L'intérêt qu'on lui porte actuellement est en nette régression; le fruit n'étant plus une base alimentaire et le bois utilisé naguère en charpente, pour les parquets, pour les piquets et échalas, la tonnellerie et pour les produits du feuillardage est aujourd'hui utilisé essentiellement pour l'extraction des tannins et la pâte à papier (de Champs, 1972; Becker et al, 1982).

Nous avons utilisé les peuplements de taillis simple de châtaignier pour f'étude des liaisons station-productionimmobilisation d'éléments nutritifs chez cette espèce (Ranger et al, 1990), mais aussi pour généraliser ces relations à l'ensemble des taillis simples.

L'objectif du travail présenté ici est : - d'apporter des données sur cette espèce qui, dans un contexte de gestion extensive d'écosystème, pourrait participer aux scénarios d'utilisation des terres libérées par l'agriculture;

- d'utiliser le modèle châtaignier pour étudier, dans les taillis simples, la dynamique d'incorporation des éléments nutritifs majeurs au cours du cycle sylvicole.

On utilise, à cette fin, une série synchrone de peuplements d'âge croissant, situés en conditions sylvicoles et écologiques identiques. 
Les résultats obtenus permettront de prendre en compte pour la gestion des écosystèmes forestiers des critères écologiques en parallèle aux critères sylvicoles plus classiquement utilisés; ces critères seront importants pour prévoir les effets de l'intensification de la sylviculture, en particulier le problème du raccourcissement de la longueur des révolutions.

\section{MATÉRIEL ET MÉTHODES}

\section{Localisation}

Nous avons utilisé l'expérience mise en place, en forêt privée, par le CRPF et le SERFOB de Poitiers (86), au lieu dit ule bois de la Taillée» sur la commune de Maisonnay (79) près de Melle.

Le climat général est de type atlantique, avec une pluviométrie moyenne annuelle de $950 \mathrm{~mm}$ et une température moyenne annuelle de $11,5^{\circ} \mathrm{C}$.

\section{Les sols}

II s'agit d'un sol à brun lessivé, mésotrophe au profil assez peu différencié, dans la définition de la CPCS (1967).

Ce sol est développé sur une formation complexe d'âge tertiaire connue sous le nom de sidérolithique du Poitou. II s'agit d'un mélange d'argiles de décalcification du Dogger et de produits détritiques d'origine continentale (Steinberg, 1967). Pendant le tertiaire, ces formations ont subi une évolution géochimique caractérisée par une hydrolyse alcaline mettant la silice en solution (celle-ci épigénise totalement la partie supérieure des calcaires) et une rubéfaction intense, comme en témoigne le taux de fer libre dont la valeur peut atteindre $5 \%$ à la base du profil, ainsi que la présence de pisolites de fer.

Les argiles sont de type illite, kaolinite et des édifices fer-argile (Steinberg, 1967).

Un épandage limoneux superficiel de faible épaisseur recouvre le plateau. Les sols sont polygéniques et polyphasés.
Le tableau I donne l'analyse d'un profil représentatif de la parcelle expérimentale.

\section{Caractéristiques physiques}

Les analyses sommaires effectuées montrent que les sols sont bien structurés, argileux en profondeur mais restant pénétrables et aérés vraisemblablement en liaison avec le type d'argile (kaolinite). La densité du sol n'est pas très élevée (rarement supérieure à 1), le taux de cailloux (environ $30 \%$ ) diminue la tendance à la compaction.

\section{Caractéristiques chimiques}

Les sols sont acides avec un pH compris entre 4,5 et 5 ; leur taux de saturation varie de 50 à $80 \%$.

\section{Azote et $\mathrm{C} / \mathrm{N}$}

Avec un taux d'azote de $4 \%$ et un $\mathrm{C} / \mathrm{N}$ de 10 en A1, les sols sont bien pourvus et présentent une minéralisation correcte de l'azote.

\section{Phosphore}

Le sol est très bien pourvu en cet élément dans les horizons superficiels, mais aussi dans les horizons minéraux puisque la valeur moyenne pondérée sur le profil est de 0,30\%; compte tenu de la teneur en oxydes de fer, il n'est pas impossible que le pouvoir fixateur du sol pour cet élément limite la disponibilité révélée par l'analyse.

\section{Potassium}

Les teneurs sont moyennes sur l'ensemble du profil, mais plutôt faibles dans l'horizon organique.

\section{Calcium}

Avec une teneur moyenne de 3 meq/100 g sur le profil entier et d'environ $2 \mathrm{meq} / 100 \mathrm{~g}$ dans les horizons organiques, ce sol est très bien pourvu en $\mathrm{Ca}$.

\section{Magnésium}

Les teneurs moyennes supérieures à $1,5 \mathrm{meq} /$ $100 \mathrm{~g}$ sur le profil entier et à $1 \mathrm{meq} / 100 \mathrm{~g}$ dans les horizons organiques montrent que ce sol est très bien pourvu en cet élément.

Au total, ce sol est bien pourvu en éléments nutritifs, sans problèmes physiques particuliers 
Tableau I. Quelques caractéristiques des sols de l'expérience de Melle. Données du peuplement de 19 ans.

\begin{tabular}{|c|c|c|c|c|c|}
\hline \multirow{2}{*}{$\begin{array}{l}\text { Caractéristiques } \\
\text { des sols }\end{array}$} & \multicolumn{5}{|c|}{ Horizons } \\
\hline & $A$ & $A(B)$ & $\|(B 1)$ & $I(B 2)$ & $I I(B) / C$ \\
\hline $\begin{array}{l}\text { Profondeur }(\mathrm{cm}) \\
\mathrm{da} \\
\mathrm{pH}\end{array}$ & $\begin{array}{c}0-10 \\
0,60 \\
4,95\end{array}$ & $\begin{array}{c}10-22 \\
0,96 \\
4,60\end{array}$ & $\begin{array}{c}22-42 \\
0,94 \\
4,65\end{array}$ & $\begin{array}{c}42-67 \\
0,80 \\
4,80\end{array}$ & $\begin{array}{l}>70 \\
0,71 \\
5,00\end{array}$ \\
\hline $\begin{array}{l}\text { Granulométrie } \\
\text { A } \\
\text { L } \\
\text { S }\end{array}$ & $\begin{array}{l}22,3 \\
65,2 \\
12,5\end{array}$ & $\begin{array}{l}24,2 \\
59,2 \\
16,6\end{array}$ & $\begin{array}{r}51,8 \\
39,6 \\
8,6\end{array}$ & $\begin{array}{r}64,3 \\
27,0 \\
8,7\end{array}$ & $\begin{array}{r}69,8 \\
23,2 \\
7,0\end{array}$ \\
\hline $\begin{array}{l}\text { Cailloux (\%) } \\
\mathrm{C}(\%) \\
\mathrm{N}(\% \circ) \\
\mathrm{C} / \mathrm{N} \\
\mathrm{P}_{2} \mathrm{O}_{5}(\% \circ)\end{array}$ & $\begin{array}{r}14,5 \\
4,42 \\
4,20 \\
10,52 \\
0,24\end{array}$ & $\begin{array}{c}35,0 \\
1,06 \\
1,21 \\
8,75 \\
0,21\end{array}$ & $\begin{array}{c}21,5 \\
0,60 \\
0,81 \\
7,41 \\
0,34\end{array}$ & $\begin{array}{c}17,0 \\
0,38 \\
0,78 \\
4,90 \\
0,43\end{array}$ & $\begin{array}{l}22,0 \\
- \\
- \\
- \\
0,34\end{array}$ \\
\hline $\begin{array}{l}\text { Eléments libres } \\
\text { Al } \\
\mathrm{Fe}\end{array}$ & $\begin{array}{l}0,38 \\
2,13\end{array}$ & $\begin{array}{l}0,43 \\
2,76\end{array}$ & $\begin{array}{l}0,57 \\
3,85\end{array}$ & $\begin{array}{l}0,70 \\
4,31\end{array}$ & $\begin{array}{l}0,64 \\
4,71\end{array}$ \\
\hline $\begin{array}{l}\text { Eléments échang } \\
\text { (meq/100 g) } \\
\mathrm{K} \\
\mathrm{Ca} \\
\mathrm{Mg} \\
\mathrm{Mn} \\
\mathrm{Na} \\
\mathrm{Ae} \\
\mathrm{H} \\
\mathrm{T} \\
(\mathrm{S} / \mathrm{T}) \times 100\end{array}$ & $\begin{array}{r}0,43 \\
3,69 \\
1,60 \\
0,84 \\
0,18 \\
0,77 \\
0,29 \\
7,79 \\
86,41\end{array}$ & $\begin{array}{r}0,20 \\
0,75 \\
0,43 \\
0,46 \\
0,09 \\
1,96 \\
0,32 \\
4,20 \\
45,58\end{array}$ & $\begin{array}{r}0,31 \\
1,50 \\
1,27 \\
0,55 \\
0,13 \\
4,33 \\
0,41 \\
8,39 \\
44,83\end{array}$ & $\begin{array}{r}0,38 \\
3,19 \\
2,30 \\
0,25 \\
0,17 \\
7,54 \\
0,46 \\
14,29\end{array}$ & $\begin{array}{r}0,41 \\
6,19 \\
3,52 \\
0,25 \\
0,26 \\
5,22 \\
0,30 \\
16,15\end{array}$ \\
\hline
\end{tabular}

et convient parfaitement au châtaignier compte tenu des exigences écologiques de cette essence (Rol et Jacamon, 1968). Le terme vernaculaire de "terre rouge à châtaignier", utilisé pour désigner ces sols, le laissait d'ailleurs explicitement prévoir.

Une des hypothèses fondamentales de cette expérience est que la variabilité entre les sols des différents peuplements est aussi faible que possible, de sorte que cette série diachronique peut être considérée comme une série chronologique.
La description des sols montre une homogénéité satisfaisante; le sol qui s'écarte le plus de la moyenne est le sol du peuplement de 9 ans. Les résultats analytiques montrent une variabilité non aléatoire liée essentiellement à l'importance de l'épandage de limon à la surface du sol. Les argiles rendent bien compte de la situation (fig 1) : les sols des peuplements $I$, II et $V$ sont bien identiques, le sol du peuplement IV est le plus argileux, celui du peuplement III, le moins argileux, avec des taux moyens respectifs de 46,$6 ; 44,2 ; 36,0 ; 60,1$ et 49,5 pour les peuplements I, II, III, IV et V. II est évident que 
ARGILE

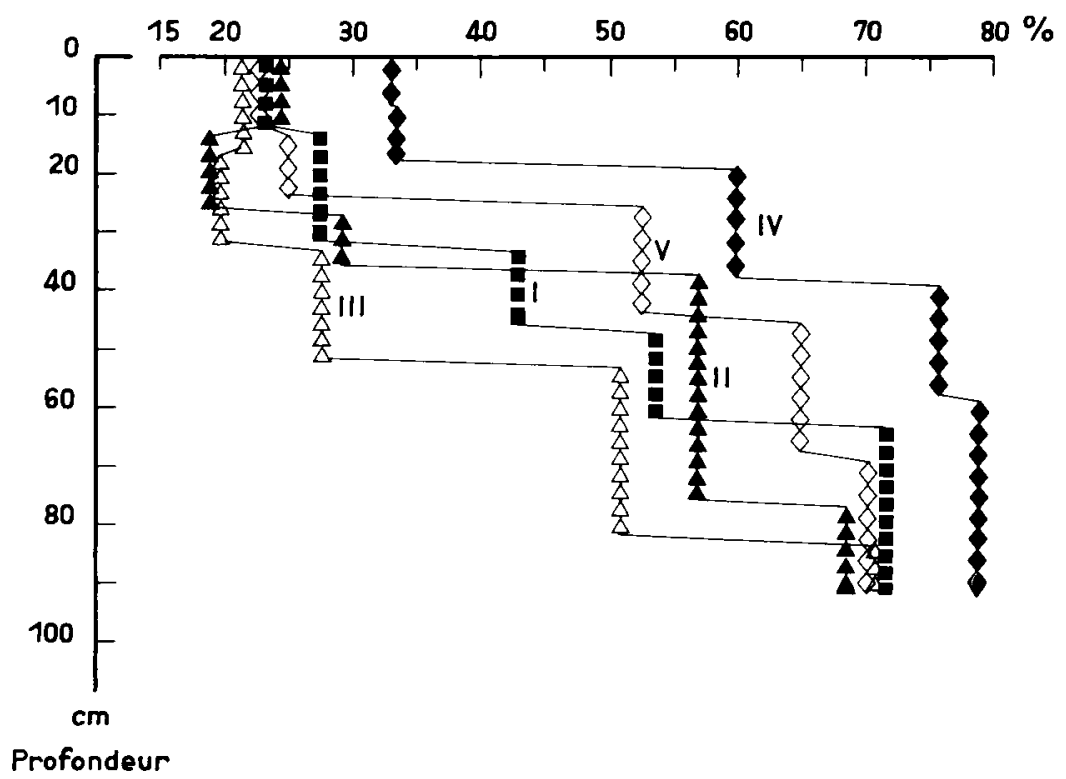

Fig 1. Distribution des argiles dans les sols des 5 peuplements.

les paramètres physico-chimiques et chimiques sont corrélés à cette variation de roche mère. Comme le montre la figure 1 , les granulométries des sols III et IV se déduisent de celles des sols des peupiements I, II ou V par une simple translation vers le bas (profil IV) ou vers le haut (profil III).

Sans nier l'importance des différences observées, il ressort que le contexte géochimique est le même et que compte tenu du niveau élevé de fertilité les différences n'ont pas l'importance qu'elles pourraient avoir dans un contexte de facteur nutritif limitant.

\section{Les peuplements}

Ils sont répartis sur la dizaine d'hectares du dispositif de sylviculture. II s'agit de 5 peuplements, dont les âges sont respectivement de 2, 5, 9, 15 et 19 ans, traités en taillis simples, pratiquement monospécifiques de châtaignier (Castanea sativa Miller); les espèces accompagnatrices peuvent représenter un nombre non négligeable de tiges, mais elles ne représentent jamais plus de $3 \%$ de la surface terrière totale. II s'agit le plus souvent de coudrier (Corylus avellana $L$ ) et secondairement du chêne (Suercus petraea Liebl).

Ces peuplements, ayant subi la même sylviculture, sont d'ensouchement ancien, sans que l'on puisse préciser davantage ce paramètre; tous ces taillis auraient au moins été recépés 5 fois. Compte tenu de la production, la rotation est fixée à 20 ans; ces taillis sont toujours exploités.

Ils sont de bonne croissance, avec un accroissement moyen annuel sur pied à 15 ans de $17 \mathrm{~m}^{3} /$ ha (partie aérienne des châtaigniers vivants arrêtée à la découpe $4 \mathrm{~cm}$ ); à 19 ans on n'a plus que $14,5 \mathrm{~m}^{3}$; cela situe ces peuplements au-dessus de la classe I de production établie par de Champs (1972) pour les taillis des régions Limousin, Languedoc et Isère. On y rencontre quelques dégâts de roulure et la présence assez systématique de "Javart", diminuant la valeur commerciale des produits; il s'agit d'un chancre fusiforme se développant à la base du tronc en liaison avec l'attaque d'un 
champignon Aldénomycète (Diplodina castanea Prill) [Lanier et al, 1976].

\section{Méthodes d'étude}

\section{Prélèvements et analyses de sol}

Une fosse pédologique a été décrite dans chaque peuplement, 4 profils supplémentaires ont été prélevés pour l'analyse (5 points d'échantillonnage au total).

Un échantillon moyen a été réalisé pour les analyses suivantes : granulométrie, fer et aluminium libres. Les analyses concernant le $\mathrm{pH}$, le carbone, l'azote, le phosphore assimilable, la capacité d'échange et les éléments échangeables au $\mathrm{pH}$ du sol, ont été réalisées sur tous les prélèvements. Les méthodes classiques utilisées sont décrites par Bonneau et Souchier (1979).

\section{Prélèvements et analyses de végétaux}

Un inventaire est réalisé sur 3 placettes de surfaces variables en fonction du nombre de tiges, à savoir, 1 a pour les peuplements de 2 à 5 ans, 2 a pour ceux de 9 à 15 ans et 3 a pour celui de 19 ans.

La méthode de prélèvement par échantillonnage proportionnel par classe de circonférence à $1,30 \mathrm{~m}\left(\mathrm{C}_{130}\right)$ et construction de tarifs de biomasse et de minéralomasse, a été utilisée. Elle a été décrite en détail par Bouchon et al (1985) et Ranger ot al (1988) pour les taillis. Vingt arbres vivants ont été prélevés par peuplement et compartimentés de la façon suivante : feuilles, branches, tronc (écorce et bois séparés) à différentes découpes $(7 \mathrm{~cm}$ et $4 \mathrm{~cm}$ au fin bout). Les arbres morts n'ont fait l'objet que d'un prélèvement général pour les 5 peuplements (10 tiges au total).

Les restitutions solides au sol par les litières ont été évaluées à partir de 20 pièges de 0,125 $\mathrm{m}^{2}$ chacun, disposés de manière systématique dans chacun des peuplements; ils ont été relevés 4 ou 5 fois par an pendant 2 ans.

Chaque compartiment de biomasse et les récoltes de litière (analysée globalement), sont échantillonnés en vue de la détermination de la matière sèche à $65^{\circ} \mathrm{C}$. Pour 15 des 20 arbres, ces échantillons sont broyés et conditionnés pour l'analyse chimique des éléments totaux majeurs ( $N, P, K, C a, M g$ ). Les protocoles utilisés ont été décrits en détail par Clément (1977).

\section{Traitement des données}

Une mise au point méthodologique, à paraître, précisera les traitements des données et la validité des résultats.

\section{Analyses statistiques}

Le traitement des données a consisté en une analyse de variance multivariable à un ou plusieurs facteurs contrôlés avec éventuellement représentation de la variabilité par l'analyse factorielle discriminante (AFD). Cette analyse a surtout été utilisée pour les paramètres qualitatifs de distribution des éléments minéraux.

\section{Les tarifs de biomasse}

La philosophie a été d'essayer d'établir des tarifs généraux pour les 5 peuplements. Les travaux de Arbonnier (1964) et de Pardé et Bouchon (1988) montrent que les tarifs montent avec l'âge. L'explication donnée par les auteurs est que les arbres, à diamètre égal, changent de forme avec l'âge, en tendant vers la cylindricité. II n'est donc pas possible d'utiliser un seul tarif pour cuber des arbres d'âges différents, sauf précisément si ce tarif a été paramétré par l'âge.

Les nuages de points, reliant la circonférence à $1,30 \mathrm{~m}\left(C_{130}\right)$ et la biomasse ou le contenu minéral d'un compartiment, ont une forme parabolique, que l'on peut aisément modéliser par l'équation:

$$
y=a_{0}+a_{1} c_{130}+a_{2} c_{130^{2}}
$$

On introduit l'âge dans le modèle en faisant l'hypothèse que, comme dans le cas général, les coefficients de cette équation varient de façon linéaire avec l'âge (Bouchon, 1974). On a ainsi l'équation générale pour l'ensemble des peuplements :

$$
\begin{gathered}
y=\beta_{0}+\beta_{1} C_{130}+\beta_{2} C_{130^{2}} \\
\text { avec : } \beta_{\mathrm{i}}=a_{\mathrm{i}}+b_{\mathrm{i}} \text { âge }
\end{gathered}
$$


On obtient ainsi le tarif paramétré par l'âge :

$$
\begin{gathered}
y=a_{0}+\underset{1}{a_{1} C_{130}+a_{2} C_{130}{ }^{2}+b_{0}} \text { âge }+b_{1} \\
\text { âge } C_{130}+b_{2} \text { âge } C_{130^{2}}
\end{gathered}
$$

Ce tarif a été testé par rapport au tarif général non paramétré par l'âge et par rapport au tarif spécifique à chaque peuplement, pour chaque élément et chaque compartiment. Les tests effectués concernent la comparaison des coefficients de corrélation, l'observation des résidus et l'ajustement du modèle aux valeurs mesurées.

Ces tarifs sont ensuite appliqués à l'ensemble des peuplements.

\section{RÉSULTATS}

\section{Distribution des éléments minéraux : aspect qualitatif}

Les différences entre les compartiments sont celles que l'on observe très classiquement; l'ordre relatif de concentrations en éléments majeurs reste le même, quel que soit l'âge du peuplement (feuilles > branches > écorce de tronc > bois de tronc). La comparaison de la concentration en bioéléments d'un même compartiment en fonction de l'âge du peuplement est plus importante, puisqu'elle pourra déterminer en partie la spécificité des tarifs, si les variations sont corrélées à l'âge. Les résultats de l'analyse de variance sont consignés dans le tableau II.

\section{Pour les feuilles}

Les différences significatives existent pour $\mathrm{N}, \mathrm{P}$ et $\mathrm{Ca}$. Elles proviennent, en fait, uniquement du peuplement de 2 ans qui montre les teneurs les plus faibles en $N, P$, $\mathrm{K}, \mathrm{Mg}$, mais au contraire les plus fortes concentrations en $\mathrm{Ca}$. On peut penser à un phénomène de dilution pour expliquer ce résultat. Les rejets montrent toujours des feuilles de taille très grande par rapport aux peuplements adultes. Ce développement se ferait à partir des réserves de la souche qui n'a peut être pas repris son rythme de prélèvement après le traumatisme récent du recépage.

\section{Pour les branches}

Aucune différence significative n'existe entre les peuplements. La liaison négative entre le diamètre $\left(D_{10}\right)$ de la branche et la concentration en tout élément, et la distribution des diamètres en fonction de l'âge devrait conduire à une concentration plus forte dans le jeune peuplement qui ne comporte que des petits diamètres. En fait, bien qu'appartenant à la relation générale diamètre-concentration, celui-ci montre au contraire un niveau de concentration plus bas que les autres, qui peut s'expliquer vraisemblablement comme pour les feuilles.

\section{Pour l'écorce de tronc}

Quand on compare entre elles les différentes découpes pour un âge donné, on constate que pour $N, P, K$, les concentrations diminuent avec la dimension de la découpe (de la découpe 0 à la découpe 7); pour $\mathrm{Ca}$, c'est l'inverse, et pour $\mathrm{Mg}$, elles restent constantes. L'âge du compartiment est manifestement en cause. Si l'on compare un même compartiment pour les différents peuplements (dans la mesure où cela a un sens, car l'âge d'un même compartiment n'est pas égal dans les différents peuplements; par exemple la découpe 4 n'a pas le même âge dans le peuplement de 9 ans et dans celui de 19 ans), on constate que des différences significatives existent, mais qu'elles sont le plus facilement interprétables pour la découpe 7. Pour celle-ci, on observe une variation continue des concentrations en éléments nutritifs 
Tableau II. Teneurs moyennes en éléments minéraux majeurs des différents compartiments de biomasse pour les 5 peuplements (résultats en \% de MS).

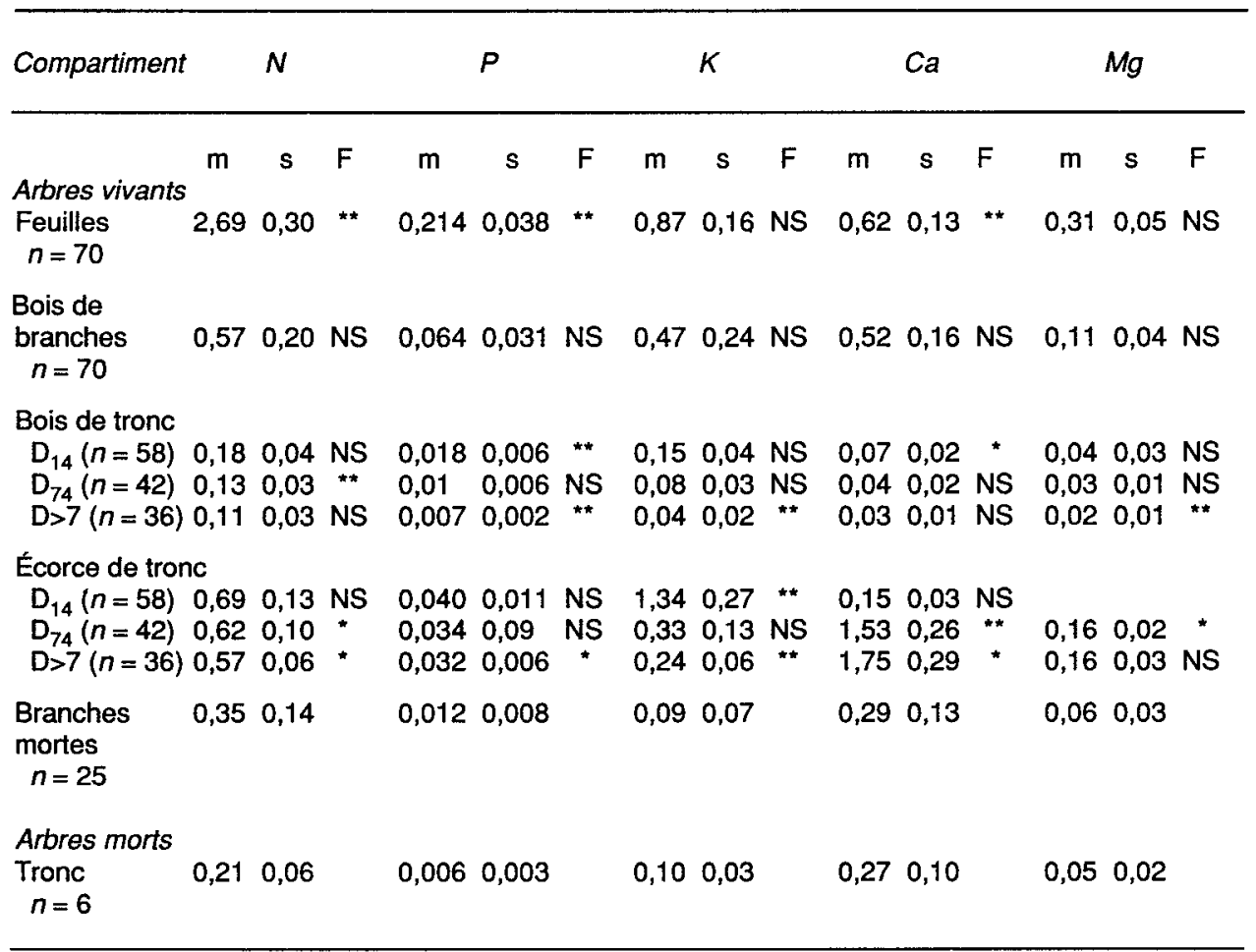

$D_{14}, D_{74}$ et $D>7$ représentent les découpes de tronc oomprises respectivement entre les valeurs de diamétre de 1 , $4,7 \mathrm{~cm}$ et plus grand que $7 \mathrm{~cm} ; m=$ moyenne, $s=$ ecart type; $F=$ test de Fisher pour la composition de moyenne, NS : non significatif, ${ }^{*}: 5 \%,{ }^{* *}: 1 \%$.

qui peut s'expliquer par la dynamique du cycle interne à la plante des transferts internes permettant une réutilisation des éléments par les organes en croissance aux dépens des organes âgés (Miller et al, 1978); ce mécanisme explique également les variations entre découpes.

\section{Pour le bois de tronc}

Les variations observées sont identiques à ce que l'on observe pour l'écorce, que ce soit pour la comparaison entre les compartiments d'un même peuplement ou pour la comparaison d'un même compartiment dans les différents peuplements.

$\mathrm{Au}$ total, les résultats montrent que les variations continues (en général des diminutions), linéaires de concentration en bioéléments d'un compartiment, en fonction de l'âge du peuplement, ne peuvent être mises en évidence que pour les compartiments qui sont constitués par l'accumulation de biomasse de tous les âges depuis 
l'origine (bois de la découpe 7). II est connu que les éléments nutritifs appartiennent à un cycle biogéochimique complexe et que ses mécanismes de fonctionnement évoluent de façon non linéaire avec l'âge (Attiwill, 1980; Miller, 1981, 1984); seul le calcium, et secondairement le magnésium, pour lesquels le peuplement est toujours dépendant du sol, compte tenu de leur cycle biochimique très limité (Switzer et Nelson, 1972; Le Goaster, 1989) suivent une variation relativement simple.

C'est un aspect important qui va déterminer en partie la validité du paramétrage par l'âge, pour la minéralomasse.

\section{Les tarifs de biomasse et de minéralomasse}

Les résultats présentés dans le tableau III montrent qu'en ce qui concerne la bio- masse, quelle que soit la découpe ou le compartiment, les tarifs parametrés par l'âge ont toujours été retenus, car ils se sont révélés être les plus satisfaisants. Cette règle souffre une exception pour le peuplement de 2 ans qui n'est jamais correctement modélisé par le tarif général; un tarif spécifique lui a été appliqué.

En ce qui concerne la minéralomasse, le paramétrage par l'âge n'est satisfaisant que pour le houppier des arbres (feuilles et bois de branches); dans les troncs, seul le calcium montre parfois une variation nette avec l'âge. Par simplification, pour les troncs, les tarifs généraux non paramétrés ont été appliqués. Plusieurs remarques doivent être faites à propos de ces résultats :

- le houppier n'a pas le caractère cumulatif qu'a le tronc d'un arbre (élagage naturel), et de ce fait, la liaison avec l'âge du peuplement s'exprime plus simplement que pour les troncs;

Tableau III. Forme des tarifs utilisés pour l'évaluation des biomasse et minéralomasse des différents compartiments.

Compartiment

Matière sèche

Élément

Feuilles

Bois de branches

Découpe $4 \mathrm{~cm}$

(écorce et bois)

Découpe $7 \mathrm{~cm}$

(écorce et bois)

Tronc total

(écorce et bois)

Arbre total ligneux
Tarif paramétré par l'âge pour tous les éléments

Tarif paramétré par l'âge pour tous les éléments

Tarif non paramétré par l'âge
Quel que soit

le compartiment tarif paramétré par l'âge de la forme

$$
\begin{gathered}
y=a_{0}+a_{1} C_{130}+ \\
a_{2} C_{130}{ }^{2}+b_{1} \text { âge } C_{130}+ \\
b_{2} \text { âge } C_{130}{ }^{2}
\end{gathered}
$$

$$
y=a_{0}+a_{1} C_{130}{ }^{2}
$$

Tarif non paramétré par l'âge

$$
y=a_{0}+a_{1} C_{130}{ }^{2}
$$

Tarif non paramétré par l'âge

$$
y=a C_{130}^{2}
$$

Tarif non paramétré par l'âge

$$
y=a C_{130}{ }^{2}
$$


- pour les troncs, la variabilité enregistrée pour la minéralomasse, bien que faible en valeur absolue, est toujours plus élevée que pour la biomasse et peut occulter un effet âge de faible amplitude qui ne pourrait être mis en évidence que par un échantillonnage très important. L'étude qualitative des distributions montre clairement que le cas simple d'une variation continue avec l'âge est rarement vérifié, et que, par conséquent, le modèle paramétré défini précédemment ne peut être utilisé. En règle générale, les peuplements ne s'ordonnent pas de façon simple et constante quant à leur contenu minéral pour un élément donné comme c'est le cas pour la biomasse (carbone).

La figure 2 montre deux exemples illustrant les différents cas observés, le cas du tarif de la biomasse du tronc total, paramétrable par l'âge, et celui du potassium du tronc, non paramétrable par l'âge.

Compte tenu de la faible variabilité observée, le modèle général non paramétré par l'âge a été utilisé.

\section{Production de biomasse}

On voit sur la figure 3 et le tableau IV que la concurrence est très importante, notamment entre 2 et 5 ans où l'on perd près de $50 \%$ des tiges initiales et entre 5 et 9 ans où l'on perd plus de $50 \%$ des tiges res- tantes. Cette mortalité n'est pas sans influence sur les estimations de croissance en volume. Cette croissance est en effet mesurée sur les arbres restants sur pied.

La série de peuplements de Melle, permet d'estimer la mortalité. À 5 ans, on remarque qu'une grande partie des arbres morts est encore sur pied, il n'en est plus de même ultérieurement. On a donc une sous-estimation de la productivité biologique. En particulier, entre 5 et 9 ans, 18620 arbres vivants ou morts ont disparu; on peut penser que les 12900 arbres déjà morts à 5 ans en font partie. II est remarquable que pendant ces 4 années, environ 6000 arbres supplémentaires sont morts ou disparus. II n'en est plus de même ultérieurement, puisque le nombre d'arbres disparus entre 2 passages est inférieur au nombre d'arbres morts présents au début de la période.

Cependant la mortalité reste forte, comme le montre l'évolution du volume sur pied et celui de la surface terrière. À partir de 15 ans, il n'y a pratiquement plus d'augmentation de la biomasse vivante; tout se passe comme si tout nouvel accroissement de biomasse se traduisait par la mort simultanée d'une biomasse équivalente.

L'âge auquel on atteint l'acroissement maximum en surface terrière est très précoce, qu'il s'agisse de l'accroissement moyen ou de l'acroissement courant.

Tableau IV. Structure des peuplements et production en volume.

\begin{tabular}{crrrrrrr}
\hline Peuplement & $\begin{array}{c}\text { Agge } \\
\text { (ans) }\end{array}$ & $\begin{array}{c}\text { Châtaignier } \\
\text { vivants }\end{array}$ & $\begin{array}{c}\text { Divers } \\
\text { vivants }\end{array}$ & $\begin{array}{c}\text { Total } \\
\text { vivants }\end{array}$ & $\begin{array}{c}\text { Total } \\
\text { morts }\end{array}$ & $\begin{array}{c}\text { Total } \\
\text { vivants + morts }\end{array}$ & $\begin{array}{c}\text { Nombre } \\
\text { d'arbres disparus }\end{array}$ \\
\hline I & 2 & 33000 & 2350 & 35350 & 1575 & 36925 & - \\
II & 5 & 16833 & 3833 & 20666 & 12900 & 33566 & 3359 \\
III & 9 & 6417 & 1133 & 7550 & 7400 & 14950 & 18616 \\
IV & 15 & 4783 & 1767 & 6550 & 3817 & 10367 & 4583 \\
V & 19 & 2922 & 2578 & 5500 & 3111 & 8611 & 1756 \\
\hline
\end{tabular}



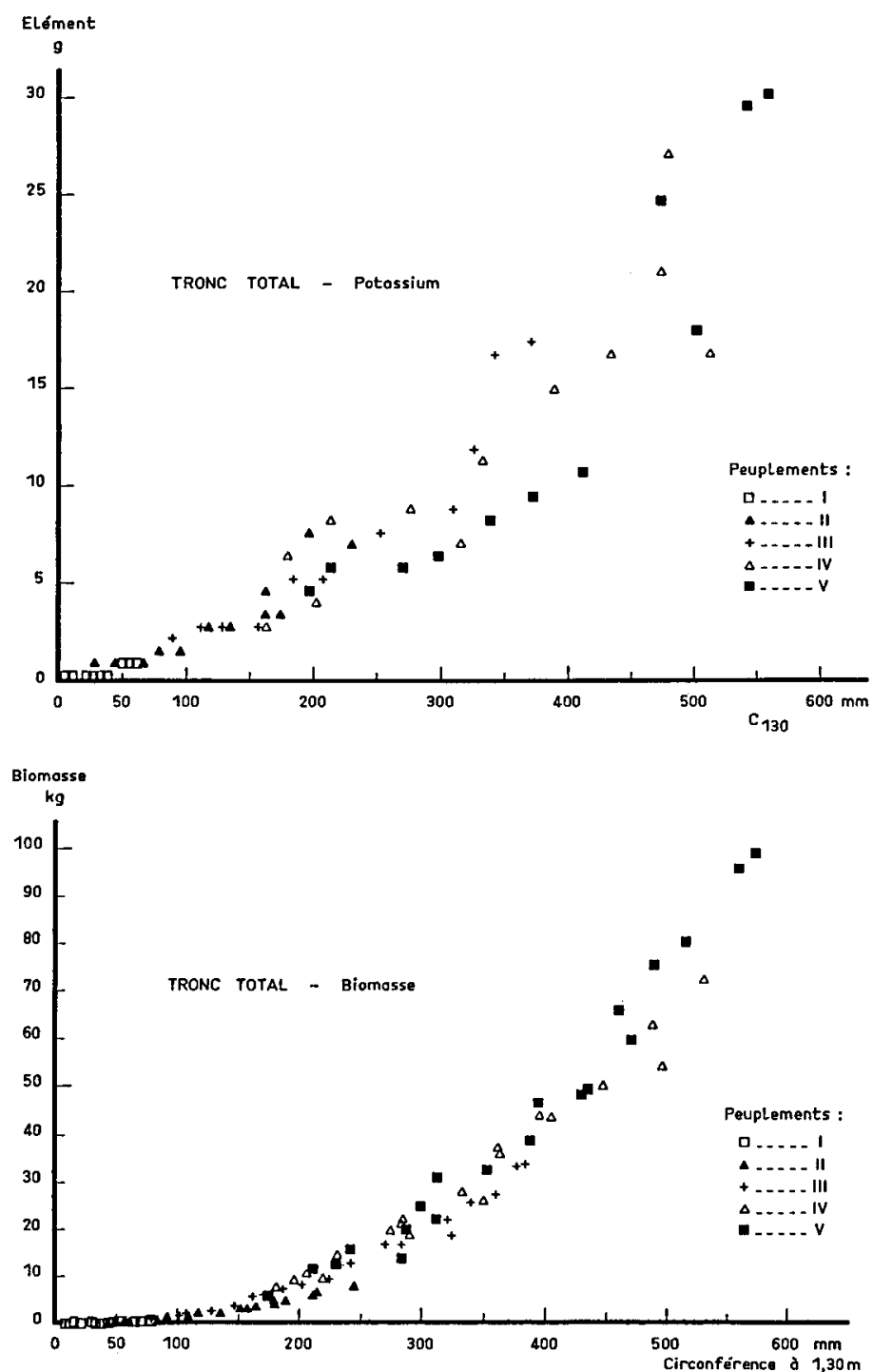

Fig 2. Relations entre la circonférence à $1,30 \mathrm{~m}$ et le contenu minéral des arbres échantillons des différents peuplements.

La croissance annuelle en hauteur est d'emblée maximale dans nos observations ( $2 \mathrm{~m}$ à 2 ans); les courbes des accroissements moyens et courants sont toujours décroissantes (fig 4A).
L'accroissement en volume montre les tendances suivantes : la valeur maximale de l'accroissement moyen annuel (volume aérien total), est pratiquement acquise dès 5 ans $\left(19 \mathrm{~m}^{3} \cdot \mathrm{ha}^{-1} \cdot \mathrm{an}^{-1}\right)$, elle évolue 


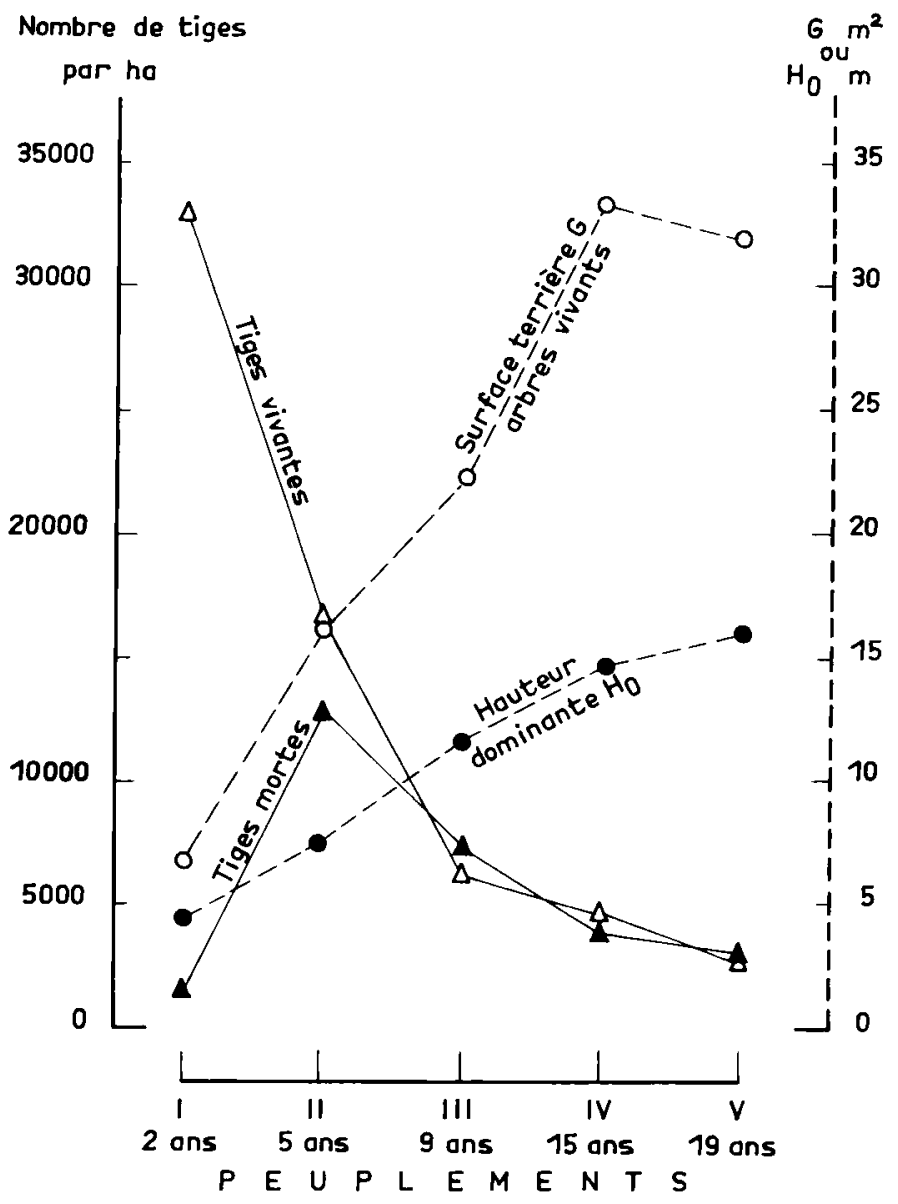

Fig 3. Structure des peuplements.

très peu par la suite, pour ce qui concerne l'accroissement courant la valeur maximale est également très rapidement atteinte (22 $\mathrm{m}^{3} \cdot \mathrm{h}^{-1} \cdot \mathrm{an}^{-1}$ à 5 ans), mais à partir de 15 ans la chute est brutale puisque l'accroissement courant passe à 8,8 $\mathrm{m}^{3} \cdot \mathrm{ha}^{-1} \cdot \mathrm{an}^{-1}$ à 19 ans (fig 4B).

L'accroissement en biomasse suit évidemment les mêmes lois avec un accroissement courant maximal dépassant $10 \mathrm{t}$ de matière sèche par ha et par an dès 5 ans. Si l'on ne tient pas compte du peuplement de 9 ans (problème de sol), on peut estimer que cette production décroît peu jusqu'à 15 ans, pour chuter ensuite très brutalement. La production moyenne totale annuelle des châtaigniers est comprise entre 6 et 7 te MS entre 5 et 19 ans. La prise en compte des essences accompagnatrices ne change rien à ces résultats puisquils sont cantonnës dans les petits diamètres et que leur biomasse totale éva- 

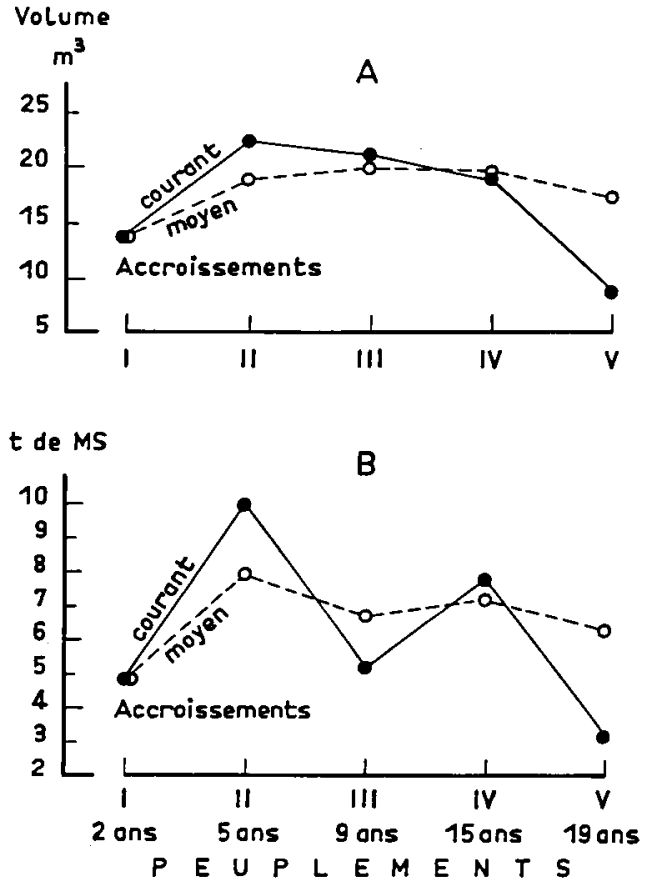

Fig 4. Accroissements moyen et courant annuels de volume et de biomasse dans les 5 peuplements.

luée par les tarifs "châtaignier» est de 0,1 ; 1,$7 ; 0,8 ; 1,6 ; 2,7$ t de MS respectivement dans les peuplements de $2,5,9,15$ et 19 ans.

La production totale se ralentit fortement dès 15 ans sur cette station riche, puisqu'en valeur absolue elle n'est que de $35 \mathrm{~m}^{3}$ entre. 15 et 19 ans. La très forte mortalité produit un changement important dans la structure du peuplement. La production de bois de diamètre moyen (découpe 4), mais surtout celle de gros bois (découpe 7), diminuent relativement beaucoup moins vite que la production totale. La production en quantité diminue (fig 5), mais fait place vers 19 ans à une production de plus grande qualité.
La production biologique réelle de ces peuplements peut être calculée avec une approximation satisfaisante, puisque l'on peut évaluer la mortalité.

Si l'on compare les performances de ces taillis aux données de la littérature (de Champs, 1972; Bouchon et al, 1985; Auclair et Métayer, 1980; Pagès, 1985; Thill, 1983; Dobremez et Merville, 1981; RuillierBréval, 1985; Chatelus, 1987; SabatierTarrago, 1989), on constate que parmi les taillis simples, les taillis de châtaignier ont la production la plus élevée, ce qui confirme les résultats de De Champs, 1972; Chatelus, 1987 et Sabatier-Tarrago, 1989, obtenus dans diverses conditions écologiques sur cette même essence.

II est d'ailleurs certain que la production de ces taillis pourrait être nettement améliorée par le renouvellement de l'ensouchement qui est, dans cette étude, toujours ancien (CRPF, communication personnelle). Riedacker (1972) a montré l'influence du nombre de rotations sur la production de taillis; dans une enquête sur l'effet du feuillardage nous avons d'ailleurs trouvé des peuplements de forte produc-

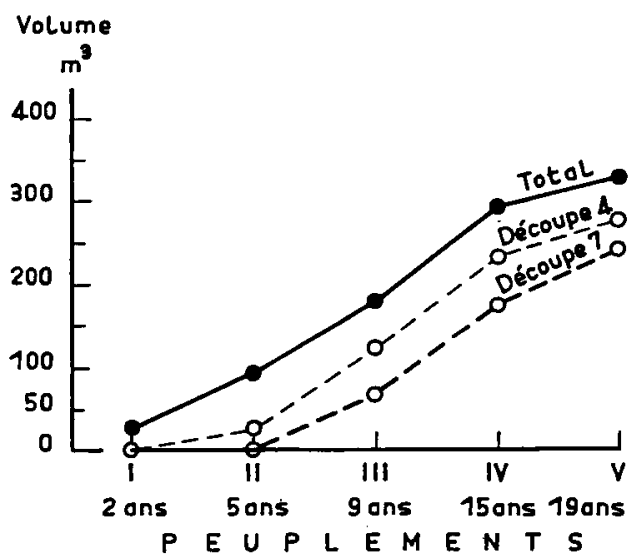

Fig 5. Volume sur pied des différents peuplements en fonction des découpes. 
tion, et la seule explication plausible du phénomène était le faible nombre de rotations (données non publiées).

\section{Incorporation d'éléments nutritifs (minéralomasse)}

La vitesse d'incorporation des bioéléments dans les peuplements, qu'il s'agisse de la vitesse moyenne ou de la vitesse courante (fig 6), montre que, dès 2 ans la vitesse maximale est atteinte pour tous les éléments majeurs. Cette vitesse maximale est stable jusqu'à 5 ans et a déjà notablement décru à 9 ans. Par opposition à la vitesse moyenne qui décroît lentement, la vitesse courante d'incorporation d'éléments nutritifs semble décroître par paliers pour passer à une valeur nulle à 19 ans.

C'est d'ailleurs quand on considère la découpe $4 \mathrm{~cm}$ (et plus encore la découpe $7 \mathrm{~cm}$ ), que la consommation courante des peuplements commence à diminuer nettement.

Le fait que le peuplement de 19 ans ait une consommation nulle par rapport au peuplement de 15 ans est lié à la disparition des petites tiges et/ou à celle des petits compartiments de biomasse les plus chargés en éléments nutrifis. En effet, les incorporations courantes d'éléments majeurs dans les découpes 4 et 7 des arbres qui restent sur pied, ne sont pas nulles (tableau V).

Ces résultats sont en accord avec ceux de Switzer et Nelson (1972) et de Madgwick et al (1980). Ils indiquent que les peuplements jeunes pour diverses raisons complémentaires, consomment beaucoup plus que les peuplements plus âgés : les tissus jeunes, sont plus riches que les tissus âgés, la proportion de tissus jeunes, actifs, est plus élevée chez les arbres jeunes, le rapport entre les compartiments
ACCROISSEMENT MOYEN ANNUEL

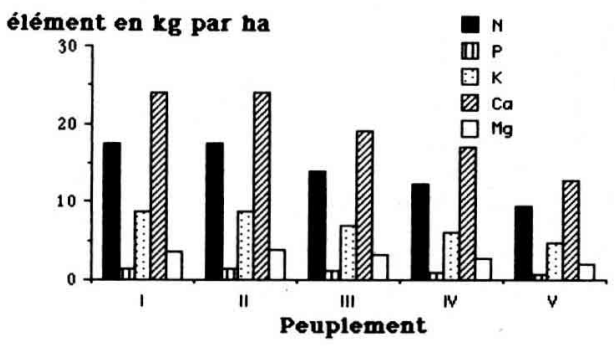

ACCROISSEMENT COURANT ANNUEL

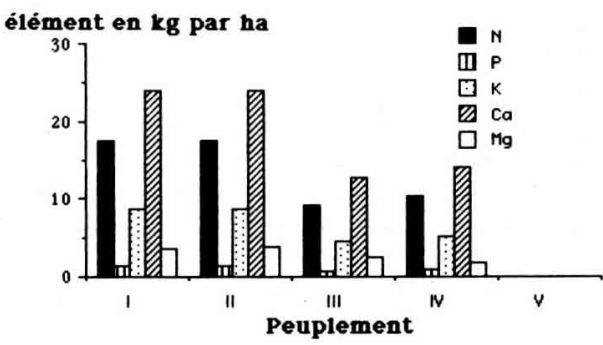

Fig 6. Accroissements moyen et courant annuels de minéralomasse des différents peuplements.

riches et les compartiments les plus pauvres est plus élevé dans les peuplements jeunes, la remobilisation des éléments des tissus âgés vers les tissus jeunes n'est pas active tant que ces tissus sont encore fonctionnels (Ranger et Bonneau, 1986).

En valeur absolue, la minéralomasse croît avec l'âge des peuplements jusqu'à 15 ans, qui stagne et régresse même à 19 ans, contrairement à la biomasse qui continue légèrement de s'accroître pendant cette période (fig 7). La majeure partie des éléments est contenue dans les petits compartiments de biomasse (tableau V). 
Tableau V. Incorporation de matière sèche et d'éléments nutritifs dans les différents peuplements (MS en t, éléments en $\mathrm{kg} / \mathrm{ha}$ ).

\begin{tabular}{|c|c|c|c|c|c|c|c|c|c|c|}
\hline & \multicolumn{2}{|c|}{$\begin{array}{c}\text { Découpe } 7 \\
\text { Bois Ecorce }\end{array}$} & \multicolumn{2}{|c|}{$\begin{array}{c}\text { Découpe } 4 \\
\text { Bois 'Ecorce }\end{array}$} & \multicolumn{2}{|c|}{$\begin{array}{l}\text { Branches } \\
\text { Bois Feuilles }\end{array}$} & \multicolumn{2}{|c|}{$\begin{array}{l}\text { Tronc total } \\
\text { Bois Écorce }\end{array}$} & Total & $\begin{array}{c}\text { Total arbre } \\
\text { tronc + } \\
\text { branches }\end{array}$ \\
\hline $\begin{array}{l}\mathrm{MS} \\
1 \\
2 \\
3 \\
4 \\
5\end{array}$ & $\begin{array}{c}0 \\
0 \\
29,9 \\
69,1 \\
89,5\end{array}$ & $\begin{array}{l}0 \\
0 \\
3,1 \\
7,6 \\
9,1\end{array}$ & $\begin{array}{r}0 \\
4,6 \\
43,1 \\
88,3 \\
100,6\end{array}$ & $\begin{array}{r}0 \\
1,6 \\
5,4 \\
10,7 \\
11,0\end{array}$ & $\begin{array}{c}0,3 \\
6,4 \\
7,7 \\
9,91 \\
10,0\end{array}$ & $\begin{array}{l}4,0 \\
4,2 \\
3,5 \\
3,2 \\
2,4\end{array}$ & $\begin{array}{r}6,4^{*} \\
25,3 \\
47,2 \\
91,2 \\
101,9\end{array}$ & $\begin{array}{r}1,3 \\
3,8 \\
6,6 \\
11,6 \\
11,5\end{array}$ & $\begin{array}{r}8,2^{*} \\
29,2 \\
54,0 \\
103,1 \\
113,7\end{array}$ & $\begin{array}{r}9,7^{*} \\
39,7 \\
60,5 \\
107,2 \\
119,9\end{array}$ \\
\hline $\begin{array}{l}N \\
1 \\
2 \\
3 \\
4 \\
5\end{array}$ & $\begin{array}{c}0 \\
0,9 \\
32,6 \\
63,8 \\
68,5\end{array}$ & $\begin{array}{c}0 \\
0,5 \\
20,4 \\
41,8 \\
44,3\end{array}$ & $\begin{array}{c}0 \\
5,1 \\
44,1 \\
85,8 \\
88,7\end{array}$ & $\begin{array}{c}0 \\
17,0 \\
32,6 \\
53,1 \\
52,2\end{array}$ & $\begin{array}{r}6,1 \\
19,4 \\
22,9 \\
30,1 \\
31,4\end{array}$ & $\begin{array}{l}82,5 \\
92,9 \\
90,7 \\
89,2 \\
68,6\end{array}$ & $\begin{array}{l}16,4 \\
37,5 \\
58,0 \\
86,9 \\
82,7\end{array}$ & $\begin{array}{l}10,9 \\
27,2 \\
38,7 \\
58,0 \\
55,2\end{array}$ & $\begin{array}{r}27,3 \\
68,0 \\
96,6 \\
144,8 \\
137,8\end{array}$ & $\begin{array}{r}35,0 \\
87,2 \\
123,9 \\
185,7 \\
176,7\end{array}$ \\
\hline $\begin{array}{l}P \\
1 \\
2 \\
3 \\
4 \\
5\end{array}$ & $\begin{array}{l}0 \\
0,02 \\
1,9 \\
4,8 \\
5,5\end{array}$ & $\begin{array}{l}0 \\
0,02 \\
1,04 \\
2,20 \\
2,36\end{array}$ & $\begin{array}{l}0 \\
0,4 \\
3,1 \\
6,0 \\
6,2\end{array}$ & $\begin{array}{l}0 \\
0,8 \\
1,7 \\
2,8 \\
2,8\end{array}$ & $\begin{array}{l}1,1 \\
2,2 \\
2,2 \\
3,0 \\
3,4\end{array}$ & $\begin{array}{l}5,3 \\
6,7 \\
7,6 \\
9,2 \\
8,2\end{array}$ & $\begin{array}{r}1,4 \\
3,4 \\
4,8 \\
7,3 \\
6,9\end{array}$ & $\begin{array}{l}0,6 \\
1,5 \\
2,1 \\
3,1 \\
3,0\end{array}$ & $\begin{array}{r}2,0 \\
4,9 \\
6,9 \\
10,4 \\
9,9\end{array}$ & $\begin{array}{r}2,7 \\
6,8 \\
9,6 \\
14,4 \\
13,7\end{array}$ \\
\hline $\begin{array}{l}K \\
1 \\
2 \\
3 \\
4 \\
5\end{array}$ & $\begin{array}{c}0 \\
0,3 \\
11,3 \\
23,5 \\
25,7\end{array}$ & $\begin{array}{r}0 \\
0,1 \\
7,2 \\
16,9 \\
18,6\end{array}$ & $\begin{array}{c}0 \\
11,9 \\
20,8 \\
33,0 \\
32,2\end{array}$ & $\begin{array}{c}0 \\
3,9 \\
12,6 \\
22,6 \\
22,8\end{array}$ & $\begin{array}{r}5,6 \\
15,9 \\
17,1 \\
22,0 \\
22,8\end{array}$ & $\begin{array}{l}30,0 \\
38,3 \\
39,1 \\
47,4 \\
44,3\end{array}$ & $\begin{array}{r}6,8 \\
16,9 \\
24,1 \\
36,1 \\
34,3\end{array}$ & $\begin{array}{r}4,9 \\
12,1 \\
17,3 \\
25,9 \\
24,6\end{array}$ & $\begin{array}{l}11,7 \\
29,1 \\
41,3 \\
61,9 \\
58,9\end{array}$ & $\begin{array}{l}17,4 \\
43,3 \\
61,5 \\
92,2 \\
87,7\end{array}$ \\
\hline $\begin{array}{l}\mathrm{Ca} \\
1 \\
2 \\
3 \\
4 \\
5\end{array}$ & $\begin{array}{c}0 \\
0,1 \\
8,8 \\
21,4 \\
24,5\end{array}$ & $\begin{array}{c}0 \\
0,7 \\
50,7 \\
124,9 \\
140,0\end{array}$ & $\begin{array}{c}0 \\
2,2 \\
14,3 \\
27,3 \\
28,2\end{array}$ & $\begin{array}{r}0 \\
9,5 \\
79,8 \\
154,9 \\
160,1\end{array}$ & $\begin{array}{r}3,0 \\
22,0 \\
24,1 \\
33,0 \\
36,3\end{array}$ & $\begin{array}{l}31,3 \\
30,7 \\
23,8 \\
18,4 \\
14,4\end{array}$ & $\begin{array}{r}5,9 \\
14,8 \\
21,0 \\
31,5 \\
29,9\end{array}$ & $\begin{array}{r}33,6 \\
83,8 \\
119,1 \\
178,6 \\
169,9\end{array}$ & $\begin{array}{r}39,6 \\
98,6 \\
140,1 \\
210,0 \\
199,9\end{array}$ & $\begin{array}{r}48,2 \\
120,0 \\
170,6 \\
255,6 \\
243,3\end{array}$ \\
\hline $\begin{array}{l}M \\
1 \\
2 \\
3 \\
4 \\
5\end{array}$ & $\begin{array}{c}0 \\
0,2 \\
6,5 \\
11,3 \\
11,6\end{array}$ & $\begin{array}{l}0 \\
0,1 \\
.5,5 \\
11,0 \\
11,5\end{array}$ & $\begin{array}{c}0 \\
6,4 \\
9,6 \\
14,4 \\
13,8\end{array}$ & $\begin{array}{c}0 \\
5,4 \\
8,9 \\
14,0 \\
13,5\end{array}$ & $\begin{array}{l}1,0 \\
4,5 \\
5,2 \\
6,6 \\
6,5 .\end{array}$ & $\begin{array}{r}13,3 \\
14,8 \\
11,9 \\
10,5 \\
7,5\end{array}$ & $\begin{array}{r}2,8 \\
7,1 \\
10,1 \\
15,0 \\
14,3\end{array}$ & $\begin{array}{r}2,8 \\
7,0 \\
9,9 \\
14,8 \\
14,1\end{array}$ & $\begin{array}{r}5,6 \\
14,0 \\
20,0 \\
29,9 \\
28,5\end{array}$ & $\begin{array}{r}7,3 \\
18,3 \\
28,3 \\
38,9 \\
37,0\end{array}$ \\
\hline
\end{tabular}

Toutes les valeurs sont issues de tarifs généraux sauf les valeurs indexées par (") issues d'un tarif spécifique.

Les conséquences sylvicoles de ces observations sont très importantes car on conçoit aisément que les très courtes rota- tions sont très défavorables au maintien de la fertilité des sols. Dans le cas présent, on observe même que la production acquise 
entre 15 et 19 ans est totalement gratuite pour l'écosystème. Elle a, certes, beaucoup chuté quantitativement, mais sa valeur économique supérieure et son coût écologique négligeable pour l'écosystème, doivent être pris en compte dans la décision de gestion de tels systèmes.

La comparaison de ces peuplements avec d'autres taillis simples extensifs ou intensifs, peut se calculer en efficience relative c'est-à-dire en quantité d'éléments nutritifs nécessaire à la production d'une unité de matière sèche (tableau VI). Les résultats montrent que le châtaignier est l'espèce la plus efficiente et pour les éléments majeurs $N, P$ et $K$, bien qu'étant l'espèce la plus productive des taillis simples extensifs; ce n'est pas le cas pour le $\mathrm{Ca}$ ou le $\mathrm{Mg}$, bien que sa performance soit aussi bonne que celle des autres essences.

\section{Évaluation des restitutions solides totales au sol, par litière et la mortalité des tiges}

Dans les plantations normalement éclaircies, les restitutions solides sont uniquement constituées de litières de feuilles et de petit bois résultant de l'élagage naturel

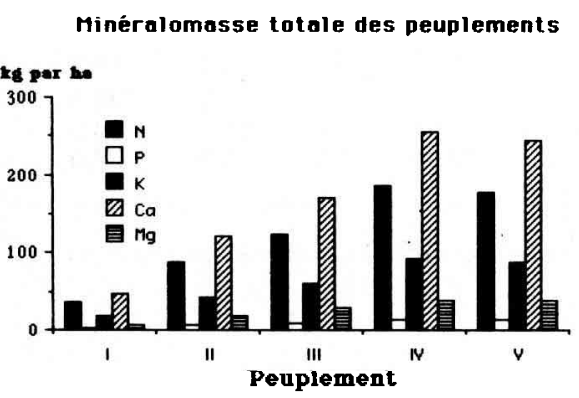

Fig 7. Minéralomasse totale des différents peuplements. des arbres (la litière). Le problème est différent dans le traitement en taillis simple où aucune récolte n'est effectuée pendant la rotation. Dans ce cas, les restitutions cumulent la litière définie ci-dessus et les tiges mortes. La production biologique totale étant la somme des tiges présentes lors de la récolte, des retombées de litière et des tiges disparues pendant la rotation.

La mobilisation d'éléments nutritifs par les peuplements et leur remise à disposition pour les arbres du peuplement finale peuvent être évaluées par ce biais.

\section{Chute annuelle de litière}

Les résultats de la figure 8 montrent qu'elle augmente rapidement jusqu'à 5 ans et qu'elle se stabilise entre 5 et 9 ans. Les récoltes effectuées pendant 2 années ne permettent pas de statistiques; la variabilité interannuelle est de l'ordre de $10 \%$ de la moyenne, et constante pour les 5 peuplements. La comparaison de ces données avec celles de la masse foliaire mesurée au même moment sur les arbres montre que la participation des bois de branches n'est pas négligeable et qu'elle augmente logiquement avec l'âge du peuplement.

Cette litière restitue une quantité non négligeable d'éléments nutritifs au sol dont la dynamique suit globablement celle de la matière sèche. À partir de 9 ans le contenu minéral est de $65 \mathrm{~kg}$ de $\mathrm{N}, 4$ à $5 \mathrm{~kg}$ de $P$, $15 \mathrm{~kg}$ de $\mathrm{K}, 40$ à 45 de $\mathrm{Ca}$, et $15 \mathrm{~kg}$ de $\mathrm{Mg}$. L'azote à tendance à baisser avec l'âge et le calcium à augmenter.

\section{Mortalité des tiges}

L'étude dynamique de la structure des peuplements permet de calculer la mortalité réelle à partir des bases de calculs suivantes: 


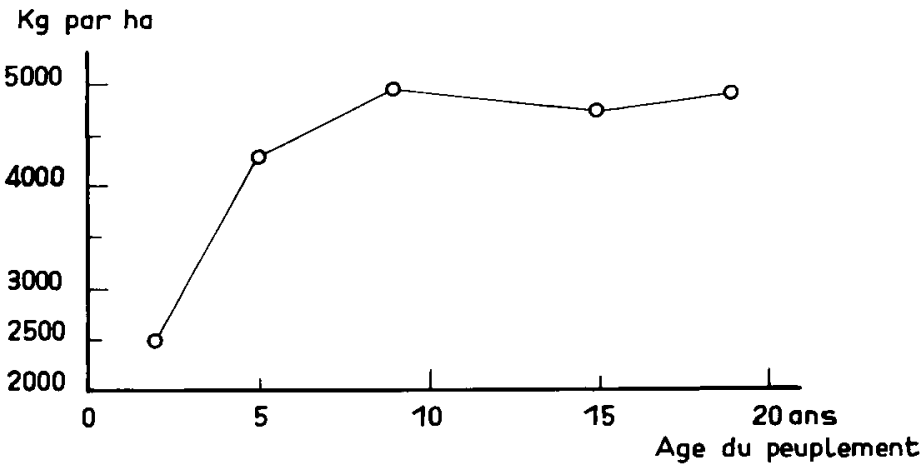

Fig 8. Restitution moyenne annuelle de litière dans les différents peuplements.

Tableau VI. Efficience de quelques essences de taillis dans l'utilisation des éléments nutritifs (résultats exprimés en kg d'élément par tonne de matière sèche ligneuse totale).

\begin{tabular}{lccccc}
\hline \multicolumn{1}{c}{ Essence } & $N$ & $P$ & $K$ & $C a$ & $M g$ \\
\hline Chêne + Bouleau & 2,54 & 0,19 & 1,04 & 1,57 & 0,23 \\
Bouchon et al, 1985 & 2,35 & 0,17 & 1,06 & 1,50 & 0,17 \\
& 2,58 & 0,26 & 1,52 & 1,95 & 0,24 \\
Peupliers & 1,17 & 0,24 & 1,37 & 5,93 & 0,38 \\
Ranger et al, 1968 & 2,51 & 0,44 & 1,59 & 7,84 & 0,29 \\
& 2,35 & 0,48 & 1,63 & 8,15 & 0,29 \\
& 2,73 & 0,46 & 1,69 & 7,50 & 0,33 \\
Châtaignier & 1,17 & 0,06 & 0,54 & 1,59 & 0,33 \\
Ranger et al, 1990 & 1,42 & 0,08 & 0,71 & 1,21 & 0,44 \\
& 1,55 & 0,08 & 0,79 & 1,66 & 0,34 \\
& 1,19 & 0,07 & 0,55 & 2,11 & 0,49 \\
& 1,36 & 0,08 & 0,64 & 1,49 & 0,40 \\
& 1,60 & 0,15 & 0,80 & 1,69 & 0,42 \\
& 1,42 & 0,08 & 0,74 & 1,69 & 0,41 \\
Châtaignier & & & & & \\
présente étude & taillis de 19 ans & 0,08 & 0,66 & 1,91 & 0,28 \\
& 1,42 & & & & \\
\hline
\end{tabular}

- chaque peuplement représente à un âge donné le peuplement final tel qu'on l'observe;
- la nécromasse disparue est déterminée à partir de l'application aux inventaires des mêmes tarifs que la nécromasse sur pied. 
Les résultats indiquent que, malgré un pas de temps de 4-5 ans entre les stades de développement, une bonne partie de nécromasse n'est pas prise en compte dans les inventaires et en particulier dans les stades initiaux.

Pour chaque stade, nous avons calculé les restitutions solides totales à la litière (puis au sol après minéralisation), depuis l'origine du peuplement (tableau VII). Si I'on considère le peuplement de 19 ans (correspondant à l'âge d'exploitabilité), on constate que $166 \mathrm{t}$ de matière sèche contenant $1400 \mathrm{~kg}$ de $\mathrm{N}, 160 \mathrm{~kg}$ de $P, 370$ $\mathrm{kg}$ de $\mathrm{K}$ et $1000 \mathrm{~kg}$ de $\mathrm{Ca}$ sont restituées au sol pendant la rotation. On peut rapprocher ces données des $120 \mathrm{t}$ de biomasse sur pied qui ne contiennent que $177 \mathrm{~kg}$ de N, $14 \mathrm{~kg}$ de $\mathrm{P}, 88 \mathrm{~kg}$ de $\mathrm{K}$ et $243 \mathrm{~kg}$ de Ca.

Ces données montrent clairement l'efficacité du cycle biologique dans la mobilisation et la mise à disponibilité d'un pool suffisant d'éléments nutritifs pour la nutrition du peuplement.

Tableau VII. Apports totaux cumulés au sol de matière sèche et d'éléments nutritifs, en fonction de l'áge des peuplements (données en $\mathrm{kg}$ par ha).

\begin{tabular}{|c|c|c|c|c|c|c|}
\hline Peuplement & Matière sèche & $N$ & $P$ & $K$ & $\mathrm{Ca}$ & $M g$ \\
\hline \multicolumn{7}{|l|}{ I (2 ans) } \\
\hline Litière* & 4997 & 58 & 3,36 & 14,2 & 5,7 & 13 \\
\hline Nécromasse ${ }^{\star *}$ & 32 & 0,09 & 0,03 & 0,03 & 0,12 & 0,02 \\
\hline Total & 5029 & 58,1 & 3,4 & 14,2 & 5,7 & 13,0 \\
\hline \multicolumn{7}{|l|}{ II (5 ans) } \\
\hline Litiere & 17894 & 242,8 & 14,9 & 50,9 & 139,2 & 47,8 \\
\hline Nécromasse & 2378 & 7,3 & 2,7 & 2,6 & 9,3 & 1,8 \\
\hline Total & 20272 & 250,1 & 17,6 & 53,5 & 148,5 & 49,6 \\
\hline \multicolumn{7}{|l|}{ II (9 ans) } \\
\hline Litière & 37706 & 544,6 & 32,5 & 121,1 & 308,8 & 106,6 \\
\hline Nécromasse & 21471 & 61,1 & 22,9 & 24,2 & 43,3 & 14,2 \\
\hline Total & 59177 & 605,7 & 55,4 & 145,3 & 352,1 & 120,8 \\
\hline \multicolumn{7}{|l|}{ IV (15 ans) } \\
\hline Litière & 66109 & 933,0 & 62,8 & 207,9 & 572,8 & 182,8 \\
\hline Nécromasse & 50318 & 138,8 & 51,3 & 57,0 & 142,6 & 32,0 \\
\hline Total & 116427 & 1071,8 & 114,1 & 264,9 & 715,4 & 214,8 \\
\hline \multicolumn{7}{|l|}{$V(19$ ans) } \\
\hline Litière & 85732 & 1179,7 & 81,2 & 276,0 & 761,2 & 232,0 \\
\hline Nécromasse & 79844 & 215,6 & 79,3 & 89,9 & 241,9 & 49,8 \\
\hline Total & 165576 & 1395,3 & 160,5 & 365,9 & 1003,1 & 281,8 \\
\hline
\end{tabular}

- La litière est calculée à partir de retombées annuelles mesurées pour chaque âge.

** La nécromasse est évaluée à partir des inventaires successifs et des tarifs établis pour les arbres morts, sur pied. 


\section{Litière au sol}

On peut considerer que, dans ce traitement et dans ce type de station, la litière disparaît totalement à chaque coupe pour se reconstituer et atteindre une masse de 15 t de MS à 15 ans. $A 19$ ans, elle retient $155 \mathrm{~kg}$ de $\mathrm{N}, 11 \mathrm{~kg}$ de $P, 22 \mathrm{~kg}$ de $\mathrm{K}, 97 \mathrm{~kg}$ de $\mathrm{Ca}$ et $24 \mathrm{~kg}$ de $\mathrm{Mg}$, ce qui ne représente que $11 \%$ de $N, 7 \%$ de $P, 6 \%$ de $K$, $10 \%$ de $\mathrm{Ca}$ et $8 \%$ de $\mathrm{Mg}$ restitués au sol pendant la rotation complète; autrement dit $89 \%$ de $N, 93 \%$ de $P, 93 \%$ de $K, 90 \%$ de Ca et $92 \%$ de $\mathrm{Mg}$ ont été recyclés pendant la rotation. C'est donc une réelle stratégie de conservation de l'écosystème qui est ici mise en évidence par l'étude de la dynamique de fonctionnement de l'écosystème.

Les coefficients de Jenny et al (1949) et les temps moyens de résidence de la matière organique et des éléments minéraux qu'ils permettent de calculer confirment tout à fait ces observations, surtout si l'on prend en compte les apports totaux au sol tels qu'ils ont été définis précédemment. Les temps de résidence 5,6 ans pour $\mathrm{MO}$ de 8,2 ans pour $N$, de 6 ans pour $P$, de 4,6 ans pour $\mathrm{K}$, de 6,6 ans pour $\mathrm{Ca}$ et $\mathrm{Mg}$ indiquent que la vitesse de restitution au pool assimilable du sol des éléments prélevés pour l'élaboration de la biomasse annuelle est rapide.

La minéralisation quasi totale, dans cette station, de la litiere lors de la coupe représente une perte potentielle d'éléments non négligeable, puisqu'aucune végétation n'est susceptible de les prélever. II semble en effet que les rejets de première année ne s'alimentent pas à partir du sol, mais plutôt à partir des réserves du système racinaire. Dans les bilans d'écosystème, c'est un poste qu'il ne faut pas négliger de prendre en compte (Ranger et Nys, 1986).

\section{CONCLUSION}

La méthode consistant à utiliser une série de peuplements d'âge croissant, situés en conditions écologiques identiques et ayant subi des traitements sylvicoles identiques, est pratiquement le seul moyen dont on dispose pour étudier la dynamique de fonctionnement des écosystèmes; cette méthode à déjà fait ses preuves (Page, 1968; Attiwill, 1980), bien que l'homogénéité des conditions précitées soit très difficile à obtenir. Les différences entre les sols des 3 peuplements, utilisés pourtant très proches (propriété d'une vingtaine d'hectares en topographie de plaine), le montrent clairement. En fait l'objet du présent travail ces difficultés ne sont pas rédhibitoires.

Le test de paramétrage des tarifs par l'âge montre que, si l'incorporation de biomasse suit une loi assez simple continue monotone avec l'âge, ce n'est pas le cas pour les éléments minéraux. Le carbone est fixé de façon définitive alors que la majorité des éléments est constamment recyclée dans l'écosystème afin d'en optimiser l'efficience, dans des systèmes qui sont le plus souvent pauvres. On s'aperçoit que seul un élément comme le calcium, pour lequel le peuplement est toujours fortement dépendant du sol, car cet élément est peu recyclé à l'intérieur de l'arbre, suit une loi simple linéaire en fonction de l'âge du peuplement.

L'étude détaillée de la distribution des éléments sera effectuée dans ces peuplements de façon à préciser la participation des différents mécanismes de recyclage et en particulier des transferts internes dans la distribution et l'utilisation des éléments nutritifs.

Les résultats montrent que le châtaignier est une espèce à forte croissance juvénile, dont le potentiel peut être important 
puisque l'on atteint une production moyenne de $6,5 \mathrm{t}$ เha ${ }^{-1} \cdot \mathrm{an}^{-1}$ à 19 ans, dans ces taillis d'ensouchement ancien; elle s'élève même à $7,2 \mathrm{t}$ à 15 ans. Dans cette station, l'accroissement moyen maximal en biomasse est atteint à 15 ans. Entre 15 et 19 ans, la structure des peuplements évolue largement avec disparition des petites tiges et transfert de l'accroissement sur les tiges de diamètre élevé. En même temps, l'immobilisation totale d'éléments nutritits par le peuplement est nulle et même négative, ce qui signifie que la production est totalement gratuite pour l'écosystème.

Le modèle châtaignier permet de répondre à des préoccupations sylvicoles plus larges, concernant le choix de la longueur des rotations. Dans ce contexte écologique, pour un objectif de production de biomasse on doit récolter à 15 ans, c'est cependant la pratique qui va être la plus préjudiciable au maintien de la fertilité des sols, et ceci d'autant plus que le taux de récolte de la biomasse sera plus important. Le maintien sur pied de ces peuplements au-delà de 15 ans se justifie tout à fait économiquement compte tenu de la plus value apportée aux produits pendant les dernières années de la vie du peuplement.

Cette approche dynamique du système permet de mettre en évidence la stratégie d'économie des peuplements vis-à-vis du stock d'éléments nutritifs, et par là même, d'indépendance vis-à-vis du sol. À partir d'un certain âge, 2 mécanismes ont été mis en évidence : les transferts internes et la mobilisation des bioéléments fortement restitués dans ce contexte de traitement sans récolte autre qu'en fin de rotation.

La comparaison de ces taillis de châtaignier avec d'autres taillis simples montre qu'en régime sylvicole extensif (en climat tempéré), cette essence peut produire le maximum de biomasse, sans apport de fertilisant. C'est une essence à ne pas né- gliger dans l'utilisation des terres libérées par l'agriculture; l'élimination des dégâts de maladie de l'encre, du javart et de roulure, améliorerait nettement le potentiel économique de cette espèce.

\section{REMERCIEMENTS}

Cette étude n'a été possible qu'avec la collaboration du SERFOB de Poitiers; nous remercions particulièrement Mme G Sauve qui nous a permis d'effectuer nos mesures dans son dispositif expérimental de Melle (79). Les graphiques ont été réalisés par $\mathrm{E}$ Henrion.

\section{RÉFÉRENCES}

Arbonnier $P$ (1964) Construction, contrôle et possibilités d'utilisation de tarifs de cubage à double entée. Application au pin laricio de Corse en Sologne. Ann Sci For 21, 527-597

Auclair D, Métayer S (1980) Méthodologie de l'évaluation de la biomasse aérienne sur pied et de la production en biomasse des taillis. Acta CEcol CEcol Plant Vol 1, 357-377

Attiwill PM (1980) Nutrient cycling in a Eucalyptus obliqua ('Hérit) forest. IV. Nutrient uptake and nutrient return. Aust J Bot 28, 199-222

Becker M, Picard JF, Timbal J (1982) Larousse des arbres des arbustes et des arbrisseaux de l'Europe occidentale. Larousse, Paris, $331 p$

Bonneau M, Souchier B (1979) Pédologie II : constituants et propriétés des sols. Masson, Paris $459 \mathrm{p}$

Bouchon J (1974) Les tarifs de cubage. ENGREF, Nancy, $57 p+$ annexes

Bouchon J, Nys C, Ranger J (1985) Cubage, biomasse et minéralomasse : comparaison de trois taillis simples des Ardennes primaires. Acta CEcol CEcol Plant 6, 53-72

de Champs $J$ (1972) La production des taillis de châtaignier. Ann AFOCEL, 221-277

Chatelus $S$ (1987) Contribution à l'analyse du cycle biologique des éléments minéraux 
dans un écosystème forestier : cas particulier du taillis de châtaignier (Castanea sativa Mill). Thèse Univ Limoges, $151 p+$ annexes

Clément A (1977) Point 1977 sur les analyses foliaires. Doc int St Sols For, INRA, Nancy, $25 \mathrm{p}$

CPCS (1967) Classification française des sols. $87 \mathrm{p}$ ronéo

Dobremez JF, Merville V (1981) Croissance, biomasse, production chute et dégradation de feuilles de taillis de châtaignier du SE de la France. Ann Sci Univ Franche-Comté, Besançon, 2, 11-20

Jenny H, Gessel SP, Bingham FT (1949) Comparative studies of decomposition rates of organic matter in temperate and tropical regions. Soil Sci, 2, 419-432

Lanier $L$, Joly $P$, Bondoux $P$, Bellemère $A$ (1976) Mycologie et pathologie forestières. Tome II Pathologie forestière. Masson, Paris, $478 p$

Le Goaster S (1989) Influence de la croissance d'un peuplement sur le prélèvement en éléments minéraux : relations avec le dépérissement des forêts. Mémoire $3^{e}$ année ENITEF, $98 p$

Magdwick HAl, Jackson DS, Knight PJ (1977) Above ground dry matter, energy and nutrient content in an age series of Pinus radiata plantations. N Z J For Sci 7, 445-468

Miller HG (1981) Nutrient cycle in forest plantations, their changes with age and the consequence for fertilizer practice. In: Proc Austr For Nutr Works: productivity in perpetuity. 187-200

Miller HG (1984) Dynamics of nutrient cycling in plantation ecosystems. In: Nutrition of plantation forest. Acad Press, Londres, 54-78

Miller HG, Cooper JM, Miller JD, Pauline OJL (1978) Nutrient cycles in pine and their adaptation to poor soils. Can J For Res 29, 26-35

Page G (1968) Some effects of conifer crops on soil properties. Commonw For Rev, 52-62

Pagès L (1985) Les taillis de Robinier du Val de Loire : croissance, biomasse, régénération. Thèse Univ Orsay, $74 p+$ annexes

Parde J, Bouchon J (1988) Dendométrie. $2^{2}$ édition, ENGREF, $328 \mathrm{p}$
Ranger J, Bonneau M (1986) Effets prévisibles de l'intensification de la production et des récoltes sur la fertilité des sols de forêt; les effets de la sylviculture. Rev For Fr 38, 105123

Ranger J, Nys C (1986) Étude des éléments minéraux dans un taillis des Ardennes (Nord de la France) : bilan pour une rotation complète. Acta CEcol CEcol, Plant 7, 287-305

Ranger J, Barnéoud C, Nys C (1988) Production ligneuse et rétention d'éléments nutritifs par les taillis à courte rotation de peuplier «Rochester": effet de la densité d'ensouchement. Acta CEcol $_{\text {CEcol Plant 9, 245-269 }}$

Ranger J, Nys C, Bouchon J (1990) Les relations entre la fertilité du sol, la production et l'utilisation des éléments nutritifs dans les taillis de châtaignier. Acta $C_{c}$ ol $C_{E c o l}$ Plant (sous presse)

Riedacker A (1972) Physiologie des souches d'Eucalyptus camaldulensis traité en taillis au Maroc. Le problème de leur vieillissement. $\mathrm{Cr}$ 70 cong for mondial, Buenos Aires, 18681875

Rol R, Jacamon M (1968) Flore des arbres arbustes et arbrisseaux III région méditerranéenne. La Maison Rustique, Paris, $95 \mathrm{p}$

Ruillier-Bréval B (1985) Croissance d'un taillis de châtaignier après coupe. Étude au cours des trois premières années. Thèse Univ Orsay, $155 p+$ annexes.

Sabatier-Tarrago C (1989) Production de taillis de châtaignier (Castanea sativa Mill) en relation avec les conditions stationnelles. Thèse Univ Orsay, $231 \mathrm{p}+$ annexes

Steinberg M (1967) Contribution à l'étude des formations continentales du Poitou (sidérolithique des auteurs). Thèse Univ Orsay, $350 \mathrm{p}$

Switzer GL, Nelson LE (1972) Nutrient accumulation and cycling in Loblolly pine (Pinus taeda L). Plantation ecosystems: the first twenty years. Soil Sci Proc 36, 143-147

Thill A (1983) Biomasse ligneuse et équivalent calorique de deux taillis de 20 et 40 ans. In: mesures des biomasses et des accroissements forestiers. Colloque IUFRO Orléans; les colloques de l'INRA $n^{\circ} 19,199-207$ 